\title{
Soy soluble polysaccharide induces apoptosis in HCT-116 human colon cancer cells via reactive oxygen species generation
}

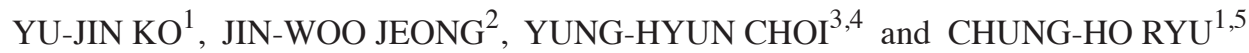 \\ ${ }^{1}$ Division of Applied Life Sciences (BK 21 plus program), Institute of Agriculture and Life Sciences, \\ Gyeongsang National University, Jinju, South Gyeongsang 660701; ${ }^{2}$ Center for Core Research Facilities, \\ Daegu Gyeongbuk Institute of Science \& Technology, Daegu, North Gyeongsang $711873 ;{ }^{3}$ Department of Biochemistry, \\ Dongeui University College of Oriental Medicine, Busan 614052; ${ }^{4}$ Anti-Aging Research Center \& Blue-Bio Industry RIC, \\ Busan 614714; ${ }^{5}$ Department of Food Science and Technology, Institute of Agriculture and Life Sciences, \\ Gyeongsang National University, Jinju, South Gyeongsang 660701, Republic of Korea
}

Received March 20, 2013; Accepted September 26, 2013

DOI: $10.3892 / \mathrm{mmr} .2013 .1725$

\begin{abstract}
Previous studies have suggested that soy sauce contains specific bioactive components and various biological activities of soy sauce have been observed. Soy soluble polysaccharide (SSPS), a predominant bioactive compound in soy sauce, has numerous pharmacological actions, including anti-inflammatory and immunomodulating activities. In the current study, the apoptotic effects of SSPS were investigated in HCT-116 human colon cancer cells. Treatment with SSPS significantly inhibited cell growth in a concentration-dependent manner by inducing apoptosis but not necrosis. This induction was associated with the generation of reactive oxygen species (ROS), mitochondrial dysfunction, activation of caspases and cleavage of the poly (ADP-ribose) polymerase protein. Induction of apoptotic cell death of HCT-116 cells by SSPS showed a correlation with the downregulation of members of the inhibitor of apoptosis protein family, including $\mathrm{X}$-linked inhibitor of apoptosis protein and antiapoptotic $\mathrm{Bcl}-2$, and upregulation of Bax and Bad. Administration of N-acetyl-L-cysteine, a scavenger of ROS, significantly decreased SSPS-induced apoptosis. These results indicate a critical role of signaling cascades involving a ROS-mediated caspase pathway in the anticancer effects of SSPS.
\end{abstract}

Correspondence to: Professor Chung-Ho Ryu, Institute of Agriculture and Life Sciences, Gyeongsang National University, 453219, 900 Gazwa-Dong, Jinju-Si, Gyeongsangnam-Do 660701, Republic of Korea

E-mail: ryu@gnu.ac.kr

Key words: soy sauce, polysaccharides, apoptosis, caspase, reactive oxygen species generation

\section{Introduction}

Apoptosis is the active process of programmed cell death, which occurs during a number of important physiological conditions and has become an area of extensive investigation in cancer research to eliminate precancerous and/or cancerous cells $(1,2)$. Apoptosis is a tightly regulated process characterized by cell shrinkage, plasma membrane blebbing and chromatin condensation that is consistent with DNA cleavage in ladders $(1,3)$. Caspase (a family of cysteine proteases) activation is often regulated by specific cellular factors, including members of the Bcl-2 family and/or the death receptor-related gene products (4). The inhibitor of the apoptosis protein (IAP) family proteins, X-linked IAP (XIAP), cellular inhibitor of apoptosis protein (cIAP)-1, cIAP-2 and survivin, by contrast, are endogenous inhibitors of apoptosis. However, the majority of cancer cells block apoptosis, which allows them to survive regardless of genetic and morphological transformations. Therefore, the induction of apoptotic cell death is an important mechanism underlying the effect of numerous anti-cancer drugs.

Reactive oxygen species (ROS) are important regulators of apoptosis. ROS production has been shown to be part of the signaling process, whereby a number of bioactive agents activate a cascade that functions to eliminate cancer cells $(5,6)$. Accordingly, excessive generation of ROS has been shown to result in cell damage and initiate certain effects (7). Although ROS are important in cell signaling, extended elevated levels of ROS lead to severe damage to DNA, RNA and proteins, which eventually results in cell death via apoptotic or necrotic mechanisms (8). In addition, ROS may act as intracellular messengers that are induced by a diverse range of stimuli and trigger apoptosis (9).

With the growth of ecological movements, natural products have become more popular for the prevention or treatment of cancer. This has been paralleled by an increase in research focused on natural products. The biological activities of the polysaccharides isolated from mushrooms, fungi, yeasts, algae, lichens and plants have attracted increased 
attention in the biochemical and medical areas due to their immunomodulatory, anticancer and antitumor effects (10). A previous study indicated cytotoxic activity of the polysaccharide against human cancer cell lines and demonstrated that the polysaccharide derived from herbal medicine may induce cell apoptosis (11). A number of bioactive polysaccharides have been isolated from botanical sources, the majority of which are relatively nontoxic with no significant side effects (12).

Soy sauce is currently used as a liquid seasoning in cooking worldwide (13) and its various biological activities have been reported, including antioxidative (14), antiplatelet (15) activities and immunomodulatory effect (16) and the inhibition of angiotensin I-converting enzyme (17). Soy soluble polysaccharides (SPS) have been reported for hypoallergenicity and antiallergic activity, and be effective for allergic disorders. Therefore, soy sauce is considered to be not only a liquid seasoning but also a functional anti-cancer food $(13,18)$. In the present study, SSPS was observed to induce a number of characteristic apoptotic symptoms in HCT-116 human colon cancer cells, including chromatin condensation, activation of caspases, cleavage of poly ADP-ribose polymerase (PARP) and mitochondrial depolarization. ROS were shown to be potential causes of the caspase activation that leads to SSPS-induced apoptosis.

\section{Materials and methods}

Reagents. 4,6-diamidino-2-phenylindole (DAPI), $\mathrm{N}$-acetyl-L-cysteine (NAC), 3-(4,5-dimethylthiazol-2-yl)-2,5-diphenyl diphenyl-tetrazolium bromide (MTT) and propidium iodide (PI) were purchased from the Sigma-Aldrich (St. Louis, MO, USA). The caspase inhibitors z-DEVD-fmk (caspase-3 inhibitor), z-IETD-fmk (caspase-8 inhibitor), z-LEHD-fmk (caspase-9 inhibitor) and 5,5',6,6'-tetrachloro-1,1',3,3'-tetraethyl-imidacarbocyanine iodide (JC-1) were purchased from Calbiochem (San Diego, CA, USA). Mouse monoclonal antibodies Bcl-2, Bad, caspase-3, actin and rabbit polyclonal antibodies against Bcl-xL, Bax, Bid, cytochrome c, caspase-8, caspase-9, XIAP, cIAP-1, cIAP-2 and PARP were purchased from Santa Cruz Biotechnology, Inc. (Santa Cruz, CA, USA). The peroxidase-labeled donkey anti-rabbit immunoglobulin and peroxidase-labeled sheep anti-mouse immunoglobulin were purchased from Amersham Pharmacia Biotech (Piscataway, NJ, USA).

Preparation of soy sauce. Fermented soybean, a seed for soy sauce, was prepared by multi-step fermentation (19). Soy sauce was fermented and aged using fermented soybean. The prepared $M e j u$ was mixed with a brine solution (20\%) at a ratio of 1:2 (w/v) and was placed in individual cylindrical mash tanks. Throughout the aging process, the temperature was maintained at $15^{\circ} \mathrm{C}$. The total aging period was three months.

IsolationandpreparationofSSPS.SSPSwerepreparedaccording to the method described previously by Kikuchi et al (20). Soy sauce $(10 \mathrm{ml})$ was dialyzed overnight using seamless cellulose tubing [width, $43 \mathrm{~mm}$; diameter $27 \mathrm{~mm}$; 12,000 MW; dialysis membrane (Viskase Companies, Inc., Darien, IL, USA)] in water at $4^{\circ} \mathrm{C}$ and freeze-dried.
Cell line, cell culture and MTT assay. HCT-116 human colon cancer cells were obtained from the American Type Culture Collection (Rockville, MD, USA) and cultured in RPMI-1640 supplemented with $10 \%$ fetal bovine serum and $1 \%$ penicillin-streptomycin at $37^{\circ} \mathrm{C}$ in a humidified chamber containing $95 \%$ air and $5 \% \mathrm{CO}_{2}$. For the cell viability assay, cells were cultured in the absence and presence of various concentrations of SSPS for $24 \mathrm{~h}$. Measurement of cell viability was determined using the MTT assay, which is based on the conversion of MTT to MTT-formazan by mitochondrial enzymes.

Flow cytometry analysis. Following treatment with SSPS, the cells were collected, washed with cold phosphate-buffered saline (PBS) and fixed in $75 \%$ ethanol at $4^{\circ} \mathrm{C}$ for $30 \mathrm{~min}$. Prior to analysis, the cells were washed once with PBS, suspended in a cold PI solution containing $100 \mu \mathrm{g} / \mathrm{ml}$ RNase A, $50 \mu \mathrm{g} / \mathrm{ml} \mathrm{PI}$, $0.1 \%(\mathrm{w} / \mathrm{v})$ sodium citrate and $0.1 \%(\mathrm{v} / \mathrm{v}) \mathrm{NP}-40$ and incubated on ice for $30 \mathrm{~min}$ in the dark. Flow cytometry analyses were conducted using a flow cytometer (FACSCalibur; BD Biosciences, San Jose, CA, USA). Cell-Quest software was used to determine the relative DNA content based on the presence of red fluorescence. The sub-G1 population was calculated to estimate the apoptotic cell population (21).

DAPI staining. Cells were washed with ice-cold PBS and fixed with $4 \%$ paraformaldehyde (Sigma-Aldrich) in PBS for 10 min at room temperature. Fixed cells were washed with PBS and stained with DAPI solution for $10 \mathrm{~min}$ at room temperature. The cells were washed twice with PBS and analyzed by fluorescence microscopy (EM-900; Carl Zeiss AG, Oberkochen, Germany).

Determination of caspase activity. The enzymatic activity of the caspases induced by SSPS was assayed using a colorimetric assay kit (R\&D Systems, Minneapolis, MN, USA) according to manufacturer's instructions. Briefly, cells were lysed in the supplied lysis buffer. The lysed cells were centrifuged at $20,000 \mathrm{x} \mathrm{g}$ for $20 \mathrm{~min}$, and equal amounts of protein $(100 \mu \mathrm{g}$ per $50 \mu \mathrm{l}$ ) were incubated with $50 \mu \mathrm{l}$ of a reaction buffer and $5 \mu \mathrm{l}$ of the colorimetric tetrapeptides, Asp-Glu-Val-Asp (DEVD)-p-nitroaniline (pNA) for caspase-3, Ile-GluThr-Asp (IETD)-pNA for caspase-8 and Leu-Glu-His-Asp (LEHD)-pNA for caspase-9, respectively. Caspase activity was determined by measuring changes in absorbance at $405 \mathrm{~nm}$ using the enzyme-linked immunosorbent assay reader.

Protein extraction and western blot analysis. Cells were harvested and lysed with lysis buffer $(20 \mathrm{mM}$ sucrose, $1 \mathrm{mM}$ EDTA, $20 \mu \mathrm{M}$ Tris-HCl, pH 7.2, $1 \mathrm{mM}$ DTT, $10 \mathrm{mM} \mathrm{KCl}$, $1.5 \mathrm{mM} \mathrm{MgCl}{ }_{2}$ and $5 \mu \mathrm{g} / \mathrm{ml}$ aprotinin) for $30 \mathrm{~min}$. The protein concentration was measured using a Bio-Rad protein assay (Bio-Rad, Hercules, CA, USA). For western blot analysis, an equal quantity of protein was subjected to electrophoresis on sodium dodecyl sulfate (SDS)-polyacrylamide gel and transferred to a nitrocellulose membrane (Schleicher \& Schuell, Inc., Keene, NH, USA). Blots were probed with the desired antibodies for $1 \mathrm{~h}$, incubated with the diluted enzyme-linked secondary antibody and visualized by enhanced chemiluminescence according to the manufacturer's instructions (Amersham Pharmacia Biotech). 
Mitochondrial membrane potential $\left(M M P, \Delta \Psi_{m}\right)$ assay. The MMP was determined using the lipophilic cationic probe JC-1. JC-1 is a radiometric, dual-emission fluorescent dye that is absorbed and concentrated by respiring mitochondria and can reflect changes in MMP in living cells. There are two excitation wavelengths, $527 \mathrm{~nm}$ (green) for the monomer form and $590 \mathrm{~nm}$ (red) for the JC-1-aggregate form. With normal mitochondrial function, MMP is high and red fluorescence is predominant. However, when there is mitochondrial injury, the MMP is reduced, leading to an increase in green fluorescence. The relative intensity of red and green fluorescent signals indicate whether mitochondria are damaged. In the current study, cells were trypsinized and cell pellets were resuspended in PBS and incubated with $10 \mu \mathrm{M} \mathrm{JC}-1$ for $20 \mathrm{~min}$ at $37^{\circ} \mathrm{C}$. Cells were analyzed using flow cytometry.

Preparation of mitochondrial proteins. Cells were treated with SSPS and harvested with ice-cold PBS. The mitochondrial and cytosolic fractions were isolated using a mitochondrial fractionation kit according to the manufacturer's instructions (Active Motif, Carlsbad, CA, USA). Cell lysates (30 $\mu \mathrm{g}$ protein/lane) were fractionated in SDS-polyacrylamide gels prior to transfer to the nitrocellulose membranes (Schleicher $\&$ Schuell) using standard instructions.

Measurement of intracellular ROS generation. Generation of ROS was determined in cells treated with SSPS in the presence and absence of NAC and was evaluated with 20,70-dichlorofluorescein diacetate (DCF-DA; Molecular Probes, Leiden, The Netherlands) as described previously (22). Cells were incubated with $10 \mu \mathrm{M}$ DCF-DA at $37^{\circ} \mathrm{C}$ for $30 \mathrm{~min}$, washed with PBS and FL-1 fluorescence was measured by flow cytometery.

Statistical analysis. The data are expressed as the mean \pm SD. A statistical comparison was performed using one-way analysis of variance followed by Fisher's exact test. The significant differences between the groups were determined using unpaired Student's t-test. $\mathrm{P}<0.05$ was considered to indicate a statistically significant difference.

\section{Results}

Inhibition of cell growth and induction of apoptosis by SSPS in HCT-116 cells. To investigate the effect of SSPS on the cell growth of HCT-116 cells, cells were exposed to specific concentrations $(0,0.3,0.6,0.9$ and $1.2 \mathrm{mg} / \mathrm{ml})$ of SSPS. Following $24 \mathrm{~h}$ incubation, the cell viability was measured using an MTT assay. As shown in Fig. 1A, treatment with SSPS significantly inhibited the viability of cells and these effects occurred in a concentration-dependent manner.

To elucidate whether SSPS inhibits the viability of HCT-116 cells by inducing apoptosis, cells treated with SSPS were examined following DAPI staining. The control cells exhibited an intact nuclear structure, whereas, cells treated with SSPS had chromosomal condensation and formation of apoptotic bodies (Fig. 1B). Morphological analysis of HCT-116 cells treated with SSPS indicated that the cells had undergone gross morphological changes indicative of
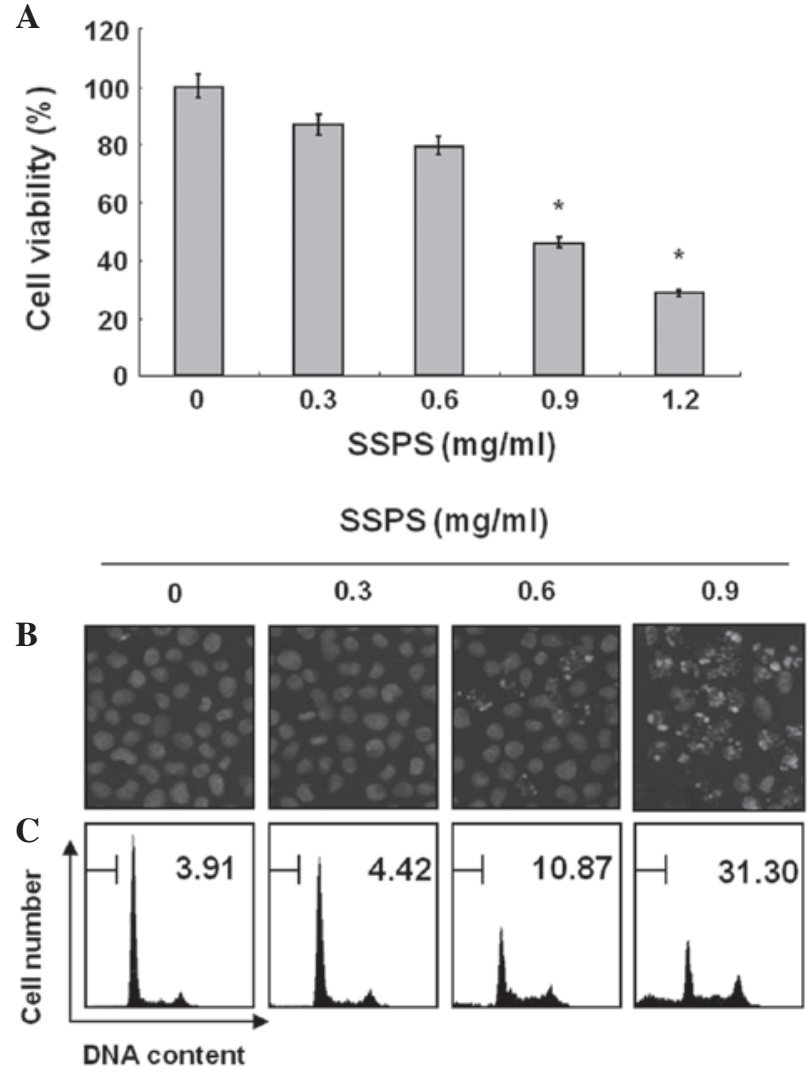

Figure 1. Inhibition of cell growth and induction of apoptosis by SSPS in HCT-116 human colon cancer cells. (A) Cells were plated at a volume of $4 \times 10^{4}$ cells per $60-\mathrm{mm}$ plate and incubated for $24 \mathrm{~h}$. Cells were treated with varying concentrations of SSPS for $24 \mathrm{~h}$ and cell viability was measured by an MTT assay. Data are expressed as the mean \pm SD of three independent experiments. For statistical analysis, Student's t-test was performed. ${ }^{*} \mathrm{P}<0.05$ vs. contol. (B) Following cell treatment with SSPS for $24 \mathrm{~h}$, cells were stained with DAPI for $10 \mathrm{~min}$ and imaged by fluorescence microscopy using a blue filter (magnification, $\mathrm{x} 400$ ). (C) Effects of SSPS on the cell cycle distribution in HCT-116 cells. Cells were incubated with specific concentrations of SSPS for $24 \mathrm{~h}$. To quantify the degree of apoptosis induced by SSPS, cells were evaluated for the sub-G1 DNA content using a flow cytometer. Data represent the means of two independent experiments. SPSS, soy soluble polysaccharide; MTT, 3-(4,5-dimethylthiazol-2-yl)-2,5-diphenyl diphenyl-tetrazolium bromide; DAPI, 4,6-diamidino-2-phenylindole.

apoptosis, including chromatin condensation and formation of apoptotic bodies.

Thus, to assess how SSPS affected cell growth, the degree of apoptosis was determined by analyzing the quantity of sub-G1 DNA in HCT-116 cells that were treated with SSPS using flow cytometry. As shown in Fig. 1C, a significant accumulation of cells with sub-G1 DNA content was noted in a dose-dependent manner, but no other markedly detectable cell cycle changes were observed in HCT-116 cells treated with the indicated concentration of SSPS for $24 \mathrm{~h}$. These results demonstrated that the cytotoxic effects observed in SSPS-treated HCT-116 cells are associated with the induction of apoptosis.

Caspase involvement in SSPS-induced apoptosis. Caspases are known to serve as important mediators of apoptosis and to contribute to the general apoptotic morphology through the cleavage of various cellular substrates, including PARP, an endogenous substrate of caspase-3 (23). Therefore, the expression levels and activities of caspase-3, -8 and -9 during SSPS-induced apoptosis were investigated. As shown in Fig. 2A, 
A

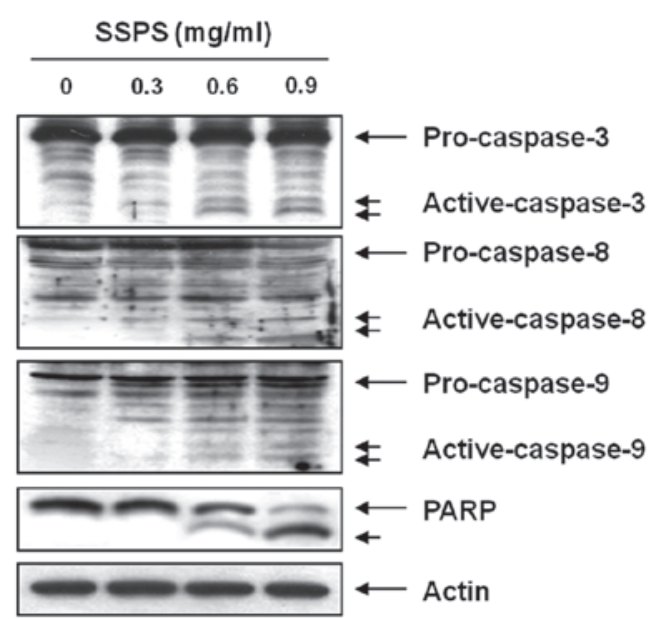

B

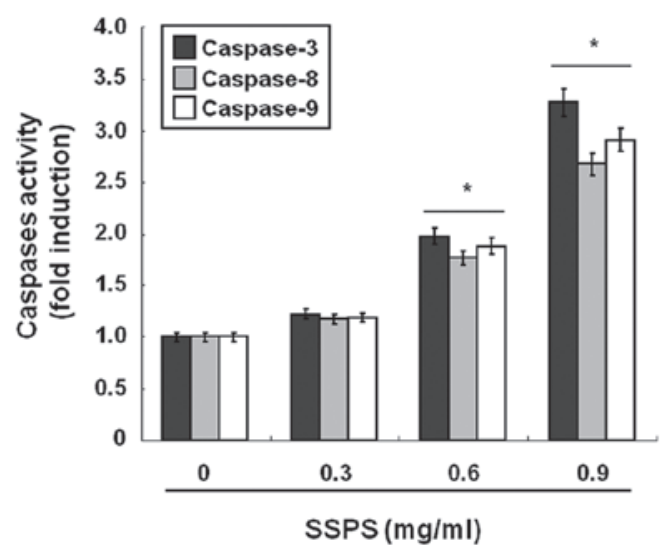

Figure 2. SSPS-induced caspase activation in HCT-116 cells. (A) Cells were treated with the indicated concentrations of SSPS for $24 \mathrm{~h}$. Equal quantities of cell lysates $(30 \mu \mathrm{g})$ were resolved by sodium dodecyl sulphate-polyacrylamide gel electrophoresis, transferred to nitrocellulose membranes and probed with antibodies against caspase- $3,-8,-9$ and PARP. Actin was used as an internal control. (B) Cell lysates obtained from cells grown under the same conditions as (A) were assayed for in vitro caspase- $3,-8$ and -9 activity using DEVD-pNA, IETD-pNA and LEHD-pNA, respectively, as substrates. The relative fluorescent products were measured. Data are presented as the mean $\pm \mathrm{SD}$ of representative experiments performed a minimum of three times. Significance was determined by Student's t-test. "P<0.05, vs. control. SPSS, soy soluble polysaccharide; pNA, p-nitroaniline; PARP, poly (ADP-ribose) polymerase.

western blot analyses showed that SSPS concentration dependently induced a marked change in the cleavage of caspase- $3,-8$ and -9 . Subsequent western blot analyses showed the progressive proteolytic cleavage of PARP in HCT-116 cells following SSPS treatment, indicating that the activation of caspase is involved in the SSPS-induced apoptotic pathway. To further quantify the proteolytic activation of caspases in HCT-116 cells, the lysates normalized for protein from the cells treated with SSPS were assayed for their activities using colorimetric assay kits. As shown in Fig. 2B, treatment with SSPS significantly increased the activities of caspase- $3,-8$ and 9 compared with control cells.

Effects of SSPS on the expression of Bcl-2 and IAP family proteins and a loss of MMP $\left({ }^{\Delta} \Psi_{m}\right)$ in HCT-116 cells. The involvement of Bcl-2 and IAP family proteins in SSPS-mediated apoptosis was determined by western blot analysis. As shown in Fig. 3A, when HCT-116 cells were treated with SSPS, a marked
A

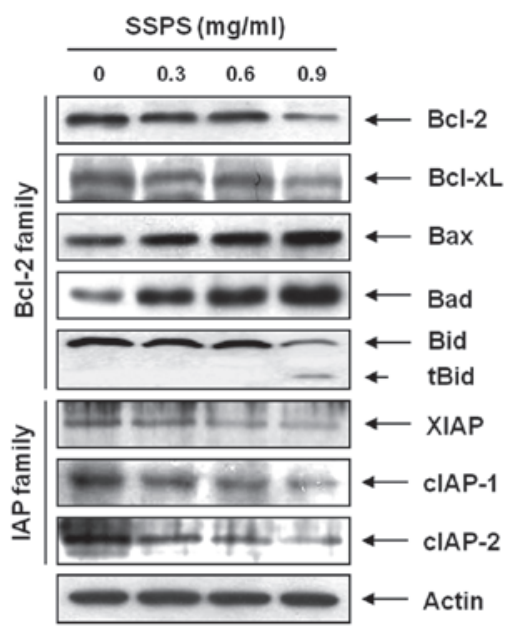

B

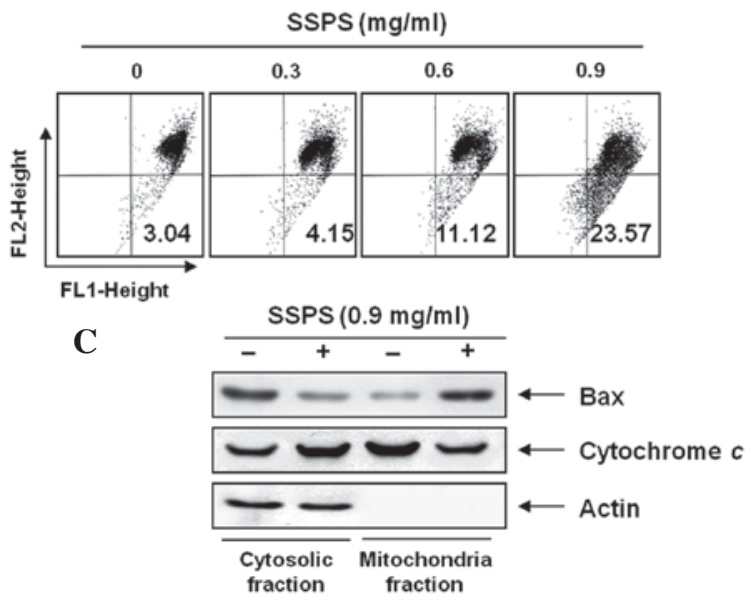

Figure 3. Effects of SSPS treatment on the levels of Bcl-2 and inhibitor of apoptosis protein family proteins, cytochrome $c$ and mitochondrial membrane hyperpolarization in HCT-116 cells. (A) Cells were treated with the indicated concentrations of SSPS for $24 \mathrm{~h}$. Proteins were detected using western blot analysis with the indicated antibodies and enhanced chemiluminescence detection. Actin was used as an internal control. (B) Cells were stained with $\mathrm{JC}-1$ for $20 \mathrm{~min}$ at $37^{\circ} \mathrm{C}$. The mean JC-1 fluorescence intensity was detected using flow cytometry. Data represent the means of two independent experiments. (C) Cytosolic and mitochondrial extracts were prepared from control and SSPS-treated cells, resolved by sodium dodecyl sulphate-polyacrylamide gel electrophoresis, transferred to nitrocellulose membranes and probed with the anti-Bax and anti-cytochrome $c$ antibodies. SPSS, soy soluble polysaccharide; JC -1, 5,5',6,6'-tetrachloro-1,1',3,3'-tetraethyl-imidacarbocyanine iodide; X-linked inhibitor of the apoptosis protein family proteins; cIAP, cellular inhibitor of apoptosis protein.

decrease in Bcl-2 and Bcl-xL antiapoptotic protein expression was observed in a concentration-dependent manner. In the case of the proapoptotic proteins Bax and Bad, there was a marginal concentration-dependent upregulation observed in HCT-116 cells treated with SSPS. In addition, western blot analyses revealed that treatment with SSPS induced the cleavage of Bid, generating a truncated form, tBid, a Bcl-2 homology domain (BH)-3-only proapoptotic member of the Bcl-2 family. The levels of XIAP, cIAP-1 and cIAP-2 were markedly inhibited by SSPS treatment, in a concentration dependent manner.

Furthermore, the involvement of the mitochondria in SSPS-induced apoptosis of HCT-116 cells was investigated by examining the effect of SSPS on the MMP values as well as the levels of cytosolic and mitochondrial Bax and cytochrome $c$. 
A Time (h)

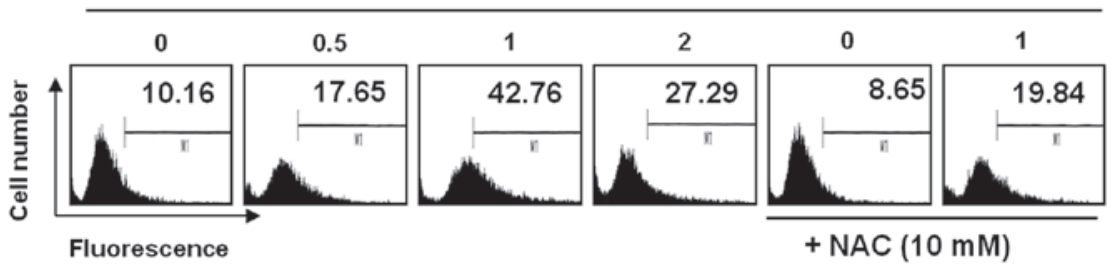

B

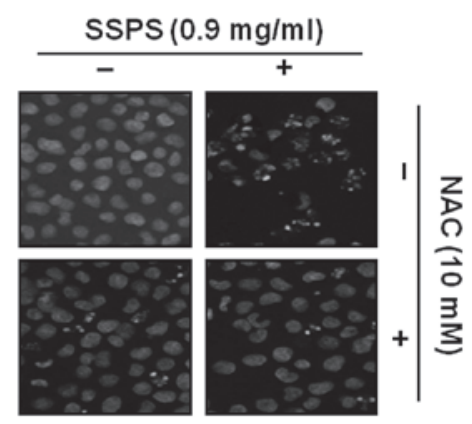

D

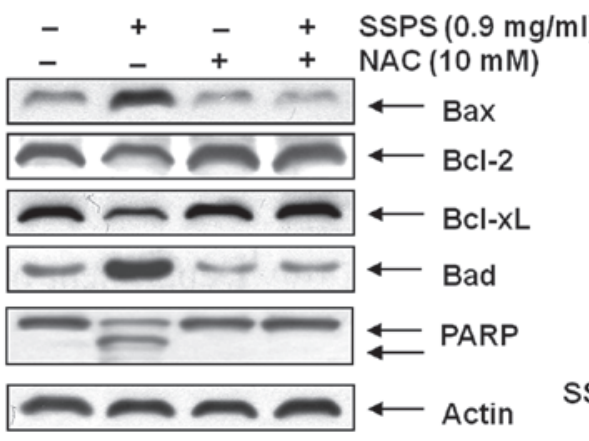

C

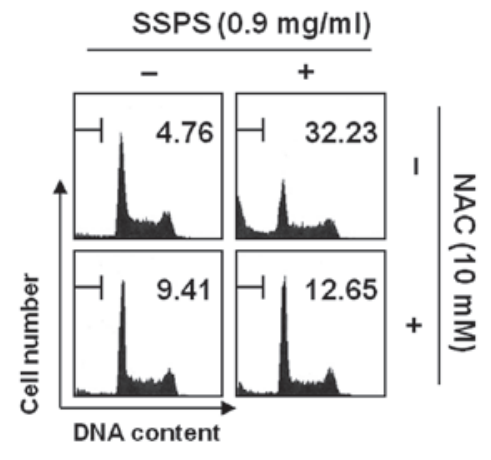

$\mathbf{E}$

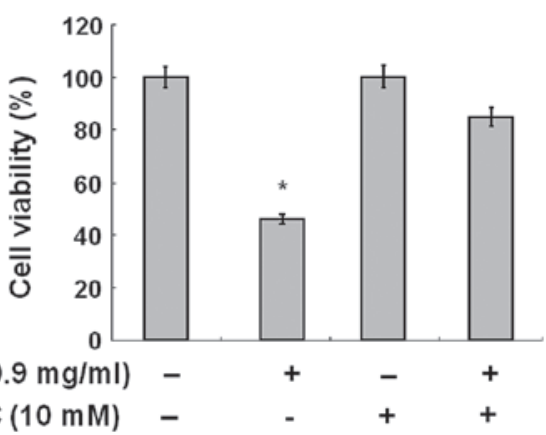

Figure 4. SSPS-induced apoptosis is associated with ROS generation in HCT-116 cells. (A) Cells were treated with or without NAC (10 mM) for $2 \mathrm{~h}$ prior to being challenged with $0.9 \mathrm{mg} / \mathrm{ml}$ SSPS for the indicated times. ROS generation was measured by flow cytometry. (B) Cells were treated with or without NAC for $1 \mathrm{~h}$ prior to being challenged with $0.9 \mathrm{mg} / \mathrm{ml}$ of SSPS for $1 \mathrm{~h}$. Following treatment with SSPS for $24 \mathrm{~h}$, cells were stained with DAPI and imaged by fluorescence microscopy using a blue filter (magnification, $\mathrm{x} 400$ ). (C) In a parallel experiment, cell cycle distribution was analyzed by flow cytometry following $24 \mathrm{~h}$ incubation. Experiments were performed three times independently, with similar results obtained in each experiment. (D) Cellular proteins extracted from cells grown under the same conditions as (B) were separated by sodium dodecyl sulphate-polyacrylamide gel electrophoresis and transferred onto nitrocellulose membranes. Membranes were probed with the indicated antibodies. Actin was used as an internal control. (E) The cells under the same conditions as (B) were evaluated for cell viability. "P $<0.05$, vs. control. SPSS, soy sauce polysaccharide; ROS, reactive oxygen species; NAC, N-acetyl-L-cysteine; DAPI, 4,6-diamidino-2-phenylindole; PARP, poly (ADP-ribose) polymerase.

As shown in Fig. 3B, exposure of HCT-116 cells to various concentrations of SSPS led to a significant reduction in the level of MMP and this reduction occurred in a dose-dependent manner. In addition, exposure of HCT-116 cells to SSPS led to a significant increase in the level of cytosolic proapoptotic protein cytochrome $c$ and to the decrease of Bax (Fig. 3C). By contrast, treatment with SSPS induced a significant downregulation of cytochrome $c$ and upregulation of Bax protein in the mitochondria. These results indicate that SSPS induced mitochondrial dysfunction in HCT-116 cells.

SSPS-induced apoptosis is associated with the generation of ROS. SSPS was investigated to determine whether it may stimulate ROS generation in HCT-116 cells. In HCT-116 cells exposed to SSPS for the indicated time periods, generation of ROS was observed at $0.5 \mathrm{~h}$ and the levels continued to increase at $1 \mathrm{~h}$ (Fig. 4A). The ROS scavenger NAC at $10 \mathrm{mM}$ significantly blocked the levels of ROS from the SSPS-treated HCT-116 cells. NAC scavenges ROS in cells by interacting with
$\mathrm{OH}^{-}$and $\mathrm{H}_{2} \mathrm{O}_{2}$ and thus affect ROS-mediated signaling pathways (24). As shown in Fig. 4B and C, pretreatment of HCT-116 cells with NAC attenuated chromatin condensation and formation of apoptotic bodies and restored the accumulation of the sub-G1 DNA population. In addition, blocking of the generation of ROS by pretreatment of the cells with NAC prevented the SSPS-induced modulation of Bcl-2 family proteins and the cleavage of PARP (Fig. 4D). Inhibiting the generation of ROS by pretreatment of the cells with NAC significantly prevented the SSPS-induced loss of cell viability (Fig. 4E).

\section{Discussion}

In 1972, Kikuchi and Yokotsuka (25) purified polysaccharides from soy sauce and investigated their properties in detail. Polysaccharides obtained from soybeans, one of the predominant raw materials of soy sauce, contained a large quantitiy of galacturonic acid and were marginally hydrolyzed by mold enzymes (20,25). Matsushita et al (16) and Kobayashi et al (26) 
reported immunomodulatory and anti-allergic activities of Shoyu polysaccharides from soy sauce; however, the cellular and molecular mechanisms responsible for the apoptotic effects of SSPS have not yet been determined in cancer cells. In the current study, the inhibition of cell viability was investigated by SSPS, and the concentration-dependent changes in the chromatin condensation of nuclei were determined using HCT-116 cells.

Apoptosis is mediated via the activation of an extrinsic (death receptor-mediated) or intrinsic (mitochondrial-mediated) pathway, regulated by caspases, death receptors and the Bcl-2 family (27). Mitochondrial dysfunction, including the loss of MMP $\left({ }^{\Delta} \Psi_{m}\right)$, which is accompanied by the alteration of $\mathrm{Bcl}-2$ family proteins (28) and release of cytochrome $c$ from the mitochondria into the cytosol is associated with apoptosis (29). Bid is a member of the BH-3-only family of proapoptotic proteins that initiate apoptotic cell death. During death receptor-induced apoptosis, Bid is activated through cleavage by caspase- 8 , generating a truncated form, tBid. $\mathrm{tBid}$ and Bax cooperate to induce mitochondrial dysfunction, including cytochrome $c$ release (23). The IAP family proteins reportedly block apoptosis due to their function as direct inhibitors that bind to and inhibit a number of caspases (30). In the current study, SSPS-induced apoptosis was shown to associate with a decrease in Bcl-2 and cleavage of Bid. Furthermore, expression levels of the XIAP proteins were decreased by SSPS (Fig. 2). Caspases are known to act as important mediators of apoptosis and to contribute to the overall apoptotic morphology via the cleavage of specific cellular substrates. In particular, activation of caspase- 3 is responsible for the proteolytic degradation of a number of key proteins, including PARP, finally leading to apoptosis (31). The present results indicate that SSPS treatment induces activation of caspase-3, -8 and -9 and concomitant proteolytic degradation and downregulation of PARP proteins.

ROS have been shown to induce specific biological processes, including apoptosis. Increased ROS induces the collapse of MMP therefore triggering a series of mitochondria-associated events, including apoptosis (8). Overproduction of ROS was observed in SSPS-treated cells and the SSPS-induced cell death was prevented by the antioxidant NAC, indicating that ROS generation is involved in the SSPS-induced apoptosis in HCT-116 cells.

In the present study, treatment with the natural substance SSPS was shown to lead to increased apoptosis in the human colon cancer cell line. The results demonstrate that SSPS may significantly induce apoptosis in HCT-116 cells through a mitochondria-mediated caspase pathway and ROS generation. The results obtained in the present study show that SSPS may contribute to the prevention or treatment of colorectal cancer.

\section{References}

1. Ghobrial IM, Witzig TE and Adjei AA: Targeting apoptosis pathways in cancer therapy. CA Cancer J Clin 55: 178-194, 2005

2. Han SI, Kim YS and Kim TH: Role of apoptotic and necrotic cell death under physiologic conditions. BMB Rep 41: 1-10, 2008.

3. Makin G and Dive C: Apoptosis and cancer chemotherapy. Trends Cell Biol 11: S22-S26, 2001.

4. Nagata S and Golstein P: The Fas death factor. Science 267: $1449-1456,1995$.

5. Ka H, Park HJ, Jung HJ, et al: Cinnamaldehyde induces apoptosis by ROS-mediated mitochondrial permeability transition in human promyelocytic leukemia HL-60 cells. Cancer Lett 196: 143-152, 2003.
6. Ueda S, Nakamura $\mathrm{H}$, Masutani $\mathrm{H}$, et al: Baicalin induces apoptosis via mitochondrial pathway as prooxidant. Mol Immunol 38: 781-791, 2002.

7. Davies KJ and Hochstein P: Ubisemiquinone radicals in liver: implications for a mitochondrial $\mathrm{Q}$ cycle in vivo. Biochem Biophys Res Commun 107: 1292-1299, 1982.

8. Fiers W, Beyaert R, Declercq W and Vandenabeele P: More than one way to die: apoptosis, necrosis and reactive oxygen damage. Oncogene 18: 7719-7730, 1999.

9. Chakraborti T, Das S, Mondal M, Roychoudhury S and Chakraborti S: Oxidant, mitochondria and calcium: an overview. Cell Signal 11: 77-85, 1999.

10. Lu X, Liu W, Wu J, et al: A polysaccharide fraction of adlay seed (Coixlachryma-jobi L.) induces apoptosis in human non-small cell lung cancer A549 cells. Biochem Biophys Res Commun 430: 846-851, 2013

11. Chow LW, Lo CS, Loo WT, Hu XC and Sham JS: Polysaccharide peptide mediates apoptosis by up-regulating p21 gene and down-regulating cyclin D1 gene. Am J Chin Med 31: 1-9, 2003.

12. Jeong SC, Yang BK, Ra KS, et al: Characteristics of anti-complementary biopolymer extracted from Coriolus versicolor. Carbohydr Polym 55: 255-263, 2004.

13. Kataoka S: Functional effects of Japanese style fermented soy sauce (shoyu) and its components. J Biosci Bioeng 100: 227-234, 2005.

14. Long LH, Kwee DC and Halliwell B: The antioxidant activities of seasonings used in Asian cooking. Powerful antioxidant activity of dark soy sauce revealed using the ABTS assay. Free Radic Res 32: 181-186, 2000.

15. Tsuchiya H, Sato M and Watanabe I: Antiplatelet activity of soy sauce as functional seasoning. J Agric Food Chem 47: 4167-4174, 1999.

16. Matsushita H, Kobayashi M, Tsukiyama R and Yamamoto K: In vitro and in vivo immunomodulating activities of Shoyu polysaccharides from soy sauce. Int J Mol Med 17: 905-909, 2006.

17. Kinoshita E, Yamakoshi J and Kikuchi M: Purification and identification of an angiotensin I-converting enzyme inhibitor from soy sauce. Biosci Biotechnol Biochem 57: 1107-1110, 1993.

18. Kobayashi M: Immunological functions of soy sauce: hypoallergenicity and antiallergic activity of soy sauce. J Biosci Bioeng 100: 144-151, 2005.

19. Lee JO, Park MH, Choi YH, Ha YL and Ryu CH: New fermentation technique for complete digestion of soybean protein. J Microbiol Biotechnol 17: 1904-1907, 2007.

20. Kikuchi T and Sugimoto H: Detailed structure of an acidic polysaccharide in soy sauce, confirmed by use of two kinds of purified pectinases. Agric Biol Chem 40: 87-92, 1975.

21. Jung EJ, Kim CW and Kim DR: Cytosolic accumulation of gammaH2 AX is associated with tropomyosin-related kinase A-induced cell death in U2OS cells. Exp Mol Med 40: 276-285, 2008.

22. Shin DY, Kim GY, Li W, et al: Implication of intracellular ROS formation, caspase-3 activation and Egr-1 induction in platycodon D-induced apoptosis of U937 human leukemia cells. Biomed Pharmacother 63: 86-94, 2009.

23. Jeong JW, Jin CY, Park C, et al: Induction of apoptosis by cordycepin via reactive oxygen species generation in human leukemia cells. Toxicol In Vitro 25: 817-824, 2011.

24. Moon DO, Kim MO, Choi YH, Hyun JW, Chang WY and Kim GY: Butein induces $\mathrm{G}(2) / \mathrm{M}$ phase arrest and apoptosis in human hepatoma cancer cells through ROS generation. Cancer Lett 288: 204-213, 2010.

25. Kikuchi T and Yokotsuka T: Studies on the polysaccharides from soy sauce Part I. Agric Biol Chem 36: 544-550, 1972.

26. Kobayashi M, Matsushita H, Yoshida K, Tsukiyama R, Sugimura T and Yamamoto K: In vitro and in vivo anti-allergic activity of soy sauce. Int J Mol Med 14: 879 -884, 2004.

27. Tsujimoto Y: Cell death regulation by the $\mathrm{Bcl}-2$ protein family in the mitochondria. J Cell Physiol 195: 158-167, 2003.

28. Kroemer G and Reed JC: Mitochondrial control of cell death. Nat Med 6: 513-519, 2000.

29. Green DR: Apoptotic pathways: ten minutes to dead. Cell 121: 671-674, 2005.

30. Gao Z, Tian Y, Wang J, et al: A dimeric Smac/diablo peptide directly relieves caspase-3 inhibition by XIAP. Dynamic and cooperative regulation of XIAP by Smac/Diablo. J Biol Chem 282: 30718-30727, 2007.

31. Lazebnik YA, Kaufmann SH, Desnoyers S, Poirier GG and Earnshaw WC: Cleavage of poly(ADP-ribose) polymerase by a proteinase with properties like ICE. Nature 371: 346-347, 1994. 